\title{
Series of magnetoresistance oscillations of a two-dimensional electron gas in a strong periodic magnetic modulation
}

\author{
Q. W. Shi and K. Y. Szeto \\ Department of Physics, The Hong Kong University of Science and Technology, Clear Water Bay, Kowloon, Hong Kong
}

(Received 11 September 1996)

\begin{abstract}
A nonperturbative technique is used to calculate the magnetoresistance of two-dimensional electron gases (2DEG) subjected to a spatially periodic magnetic field $B_{1}$ with period $a$ in addition to a uniform magnetic field $B_{0}$ in the same direction. For small amplitude modulation, magnetoresistance exhibits oscillations similar to the Weiss oscillations observed in 2DEG under spatially periodic electric field and uniform magnetic field. In general, the oscillation is characterized by two ratios: $\beta=2 \pi l / a$ and $\gamma=B_{1} / B_{0}$, at low magnetic modulations, with $l$ being the magnetic length corresponding to $B_{0}$. Quenching of Weiss oscillations first appears as $\gamma$ increases for fixed $B_{0}$, and a series of oscillations then appears in our nonperturbative calculation for larger $\gamma$. The temperature dependence of these oscillations shows that the basic mechanism of the production of these oscillations is similar to the Weiss oscillations. [S0163-1829(97)06307-8]
\end{abstract}

\section{INTRODUCTION}

A series of oscillations periodic in $1 / B_{0}\left(B_{0}\right.$ is the uniform magnetic field) have been observed in the magnetoresistance of a two-dimensional electron gas (2DEG) subjected to a weak one-dimensional (1D) periodic magnetic field. ${ }^{1,2}$ The experimental results are in good agreement with the theoretical predictions in the limit of a weak 1D magnetic modulation (modulation amplitude $\left|B_{1}\right| \ll\left|B_{0}\right|$ ) where the magnetoresistance $\rho_{x x}$ is expected to oscillate with minima appearing at magnetic fields given $\mathrm{by}^{3,4}$

$$
\frac{c}{B_{\lambda}}=\frac{e a}{2 \hbar k_{f}}(\lambda+1 / 4) .
$$

Here $\lambda=0,1, \ldots$ is an oscillation index, $\hbar k_{F}$ is the Fermi momentum, and $a$ the period of the 1D modulation. Similar to the electric case, the magnetic modulation leads to a modified energy spectrum ${ }^{3-5}$ and the degenerate Landau levels are transformed into bands of finite width. The dispersion of these Landau bands provides an additional contribution to the magnetoresistance $\rho_{x x}$, which vanishes when the bandwidth becomes zero (the so-called flatband condition). ${ }^{5}$ In contrast to Eq. (1), which is the flatband condition for magnetic modulation system (MMS), the flatband condition for weak electric modulation system (EMS) reads

$$
\frac{c}{B_{\lambda}}=\frac{e a}{2 \hbar k_{f}}(\lambda-1 / 4)
$$

with $\lambda=1,2, \ldots$. However, all of the works quoted above focused on the effects of a weak 1D modulation and the theoretical treatment uses perturbation calculation. In this paper, we study the transport properties of 2DEG's subjected to the variable amplitude of $1 \mathrm{D}$ magnetic modulation beyond the weak field regime using nonperturbative technique. We expect that behavior similar to the EMS case $^{7}$ can be found in the MMS case by increasing the strength of the modula- tion potential. Specifically, we expect to see that the amplitude of the Weiss oscillation initially increases with magnetic modulation and then quenches as the magnetic modulation becomes stronger. Furthermore, the amplitude of magnetic modulation required to quench a particular maximum or minimum at a given uniform magnetic field $B_{0}$ increases with $B_{0} \cdot{ }^{6}$ However, in our calculation for the MMS, we also find some features different from earlier studies in the EMS case, namely, we find that in even stronger magnetic modulation, after the original Weiss oscillations have been quenched, a different series of oscillations appear.

A one-dimensional magnetic modulation system can be realized experimentally by depositing a 1D array of magnetic bars onto a heterostructure consisting 2DEG. When a uniform magnetic field $B_{0}$ is applied, the magnetic bars change into local micromagnets and produce a grating magnetic field superimposed on the uniform magnetic field $B_{0}$, in which the magnitude of modulation varies with different magnetic material and can even be larger than the uniform field. ${ }^{1}$

To discuss the transport properties of $2 \mathrm{DEG}$ as a function of the amplitude of 1D magnetic modulation, we follow the ideas of Ref. 12, which uses exact diagonalization of Hamiltonion $H$ in the harmonic oscillator basis to analyze the collective excitation spectrum. However, our diagonalization is performed in Fourier space. ${ }^{7}$ We find that in Fourier space the differences between the MMS and the EMS case are more transparent. Recently, we use this nonperturbative technique to discuss the dependence of magnetoresistance $\rho_{x x}$ oscillations on the variable amplitude of 1D electric modulation, which gives good qualitative explanations of the quenching of the Weiss oscillations in a strong 1D periodic electric potential. ${ }^{7}$ We also find that this nonperturbative quantum transport calculation is more suitable than the classical theory ${ }^{9}$ which yields a good explanation of the Weiss oscillations in the weak 1D modulation but not for strong modulation. With the promising results of this nonperturbative technique for the 1D EMS case, we expect similar reliable results for our 1D MMS calculation.

In the following sections, we will first study the single 
particle electronic states of a model MMS. We consider specifically strong modulation, in order to compare this MMS case with the EMS case in a more transparent manner. We will then investigate the magnetoresistance of the MMS case and discuss the series of oscillations in strong modulation.

\section{ELECTRONIC STATES}

We consider a 2DEG system subjected to the sum of a 1D modulating magnetic field $B_{1}$ and a uniform $B_{0}$ background field, and the one-electron Hamiltonian is given by

$$
H=\frac{1}{2 m^{\star}}\left(\vec{p}+\frac{e}{c} \vec{A}\right)^{2}
$$

with $\vec{A}=\left[x B_{0}+B_{1} \sin (2 \pi x / a)\right] \hat{y}$ being the vector potential for the modulating magnetic field $\vec{B}=\left[B_{0}+B_{1} \cos (2 \pi x / a)\right] \hat{z}$ applied perpendicularly to the two-dimensional electron gas. The Hamiltonian introduces two length scales: the spatial period of the modulaton $a$ and the magnetic length $l=\left(\hbar c / e B_{0}\right)^{1 / 2}$. The system can be characterized by two dimensionless quantities: $\gamma=B_{1} / B_{0}$, the relative strength of the modulation; and $\beta=2 \pi l / a$, the relative period of modulation.

First of all, when there is no uniform magnetic field, the magnetic modulation provides a periodic potential for the two-dimensional electron gas and we have a modified Hill band structure, ${ }^{10}$ which has been studied in detail by Ibrahim and Peeters recently. ${ }^{11}$ On the other hand, when there is no modulation but the external magnetic field is present, we have the usual Landau levels with spacing $\hbar \omega_{c}$ ( $\omega_{c}=e B_{0} / m^{\star}$ and $m^{\star}$ is the effective mass). Thus, a complete solution of this problem must address the transition from the Landau level scenario to the Hill band structure ${ }^{10}$ as one turns on $B_{1}$ from zero to large values compared to $B_{0}$.

At external magnetic field $B_{0}=0.08 \sim 0.28 \mathrm{~T}$ and period spacing $a=500 \mathrm{~nm}$, the dimensionless parameter $\beta$ is about 1. Therefore, when the dimensionless parameter $\gamma$ is small, the parameter $\gamma / \beta$ can be used as a small parameter to calculate the energy spectrum and transport properties perturbatively. ${ }^{3,4}$ On the other hand, as the modulation magnetic field $B_{1}$ increases, $\gamma$ becomes large and perturbative calculation fails and we have to use some nonperturbative techniques. Since the matrix elements of the magnetic modulation terms between the eigenfunctions of harmonic oscillation can be calculated analytically, we can choose these eigenfunctions as a convenient basis for the numerical diagonalization of $H$. This is similar to the analysis of the electronic state of 2DEG in a unidirectional periodic magnetic field by $\mathrm{Wu}$ and Ulloa. ${ }^{12}$ However, our diagonalization is performed in Fourier space.

Since $k_{y}$ is a good quantum number, we can write the eigenstates as $\psi(x, y)=\exp \left(i k_{y} y\right) \psi(x)$, where $k_{y}$ is the wave vector of the electron in the $y$ direction. The wave function $\psi(x)$ satisfies the 1D Schrödinger equation:

$$
\begin{aligned}
& {\left[-\frac{\hbar^{2}}{2 m^{\star}} \frac{d^{2}}{d x^{2}}+\frac{1}{2 m^{\star}}\left(\hbar k_{y}+\frac{e B_{0}}{c}+\frac{e B_{1}}{c K} \sin K x\right)^{2}\right] \psi(x)} \\
& =E \psi(x),
\end{aligned}
$$

where $K=2 \pi / a$. We now perform the Fourier transformation on the eigenfunction of both sides of the above equation with

$$
\psi(x)=\frac{1}{2 \pi} \int d k_{x} \phi\left(k_{x}\right) \exp i k_{x}\left(x+l^{2} k_{y}\right)
$$

and obtain the delay ordinary differential equation as follows:

$$
\begin{aligned}
& \left(\frac{d^{2}}{d \xi_{x}^{2}}+\lambda-\xi_{x}^{2}\right) \phi\left(\xi_{x}\right)-(\gamma / \beta)\left[\exp \left(-i \beta \xi_{y}\right) \frac{d}{d \xi_{x}} \phi\left(\xi_{x}-\beta\right)\right. \\
& \left.\quad-\exp \left(i \beta \xi_{y}\right) \frac{d}{d \xi_{x}} \phi\left(\xi_{x}+\beta\right)\right] \\
& \quad+(\gamma / 2 \beta)^{2}\left[\exp \left(-2 i \beta \xi_{y}\right) \phi\left(\xi_{x}-2 \beta\right)\right. \\
& \left.\quad+\exp \left(2 i \beta \xi_{y}\right) \phi\left(\xi_{x}+2 \beta\right)\right]=0
\end{aligned}
$$

where $\lambda=2 E / \hbar \omega_{c}-(1 / 2)(\gamma / \beta)^{2}, \quad \xi_{x}=l k_{x}$, and $\xi_{y}=l k_{y}$. From this equation, we can separate two modulation terms: the term proportional to the parameter $\gamma / \beta$ (we call it the MMS term since this first derivative term has caused the low-field oscillations in the weak MMS), and the term proportional to the parameter $(\gamma / \beta)^{2}$ (we call it the EMS term since this term is similar to the one which has produced the Weiss oscillations in the weak EMS). Under the weak magnetic modulation, we can use the MMS term to calculate the transport properties perturbatively, and neglect the EMS term which is second order of the small parameter $\gamma / \beta$. But in the strong magnetic modulation, the perturbation method fails and the EMS term is more and more important. It is difficult to get the exact analytical solution of Eq. (6) in the general case. We therefore consider solving this equation in some limiting cases. First of all, when parameter $\beta$ is small, corresponding to a strong uniform magnetic field or to large spacing period, we can use the Taylor expansion in $\beta$ to get approximate solutions. But this is not the regime of interest here. We are interested in the magnetoresistance oscillations in the low uniform magnetic field and the interplay between the modulation $B_{1}$ and uniform field $B_{0}$.

A successful method is to calculate the numerical solution by expanding the eigenfunction $\phi$ in terms of the eigenfunction $\phi_{n}^{0}(\gamma=0)$ of the harmonic oscillator,

$$
\phi\left(\xi_{x}\right)=\sum_{n} C_{n} \phi_{n}^{0}\left(\xi_{x}\right)
$$

When we substitute Eq. (7) into Eq. (6), we obtain a series of linear algebraic equations for the expansion coefficients $C_{n}$,

$$
\begin{gathered}
\left(\lambda-\lambda_{n}\right) C_{n}-\sum_{m}\left\{\left[A U_{n m}(-\beta)-A^{\star} U_{n m}(+\beta)\right]\right. \\
\left.-\left[C V_{n m}(-2 \beta)+C^{\star} V_{n m}(+2 \beta)\right]\right\} C_{m}=0
\end{gathered}
$$




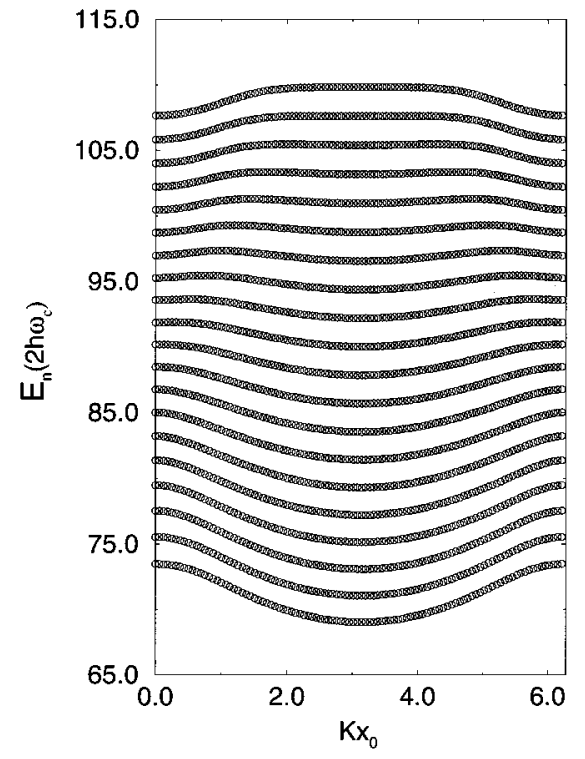

(a)

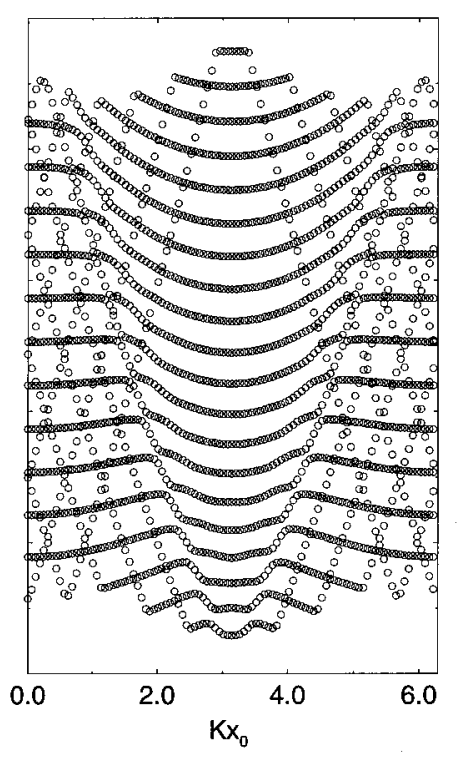

(b)
FIG. 1. Calculated energy spectrum vs center coordinate $K x_{0}=-K l^{2} k_{y}$ for $B=0.10 \mathrm{~T}$ and periodic potential with spacing $a=500 \mathrm{~nm}$. (a) A weak modulation $B_{1}=0.05 \mathrm{~T}$. (b) A strong modulation $B_{1}=0.32 \mathrm{~T}$.

$$
V_{n m}(-\beta)=\sum_{i}^{\min (m, n)} \frac{(-1)^{m-i}\left(\frac{\beta^{2}}{2}\right)^{(m+n-2 i) / 2}(m ! n !)^{1 / 2} \exp \left(-\frac{1}{4} \beta^{2}\right)}{i !(m-i) !(n-i) !}
$$

and

$$
\begin{gathered}
\lambda_{n}=2 n+1, \quad A=(\gamma / \beta) \exp \left(-i \beta \xi_{y}\right), \\
C=(\gamma / 2 \beta)^{2} \exp \left(-2 i \beta \xi_{y}\right),
\end{gathered}
$$

$A^{\star}$ and $C^{\star}$ are the complex contjugate to $A$ and $C$, respectively,

$$
V_{n m}(-\beta)=(-1)^{m+n} V_{n m}(+\beta),
$$

and

$U_{n m}( \pm 2 \beta)=\sqrt{m / 2} V_{n m-1}( \pm 2 \beta)-\sqrt{(m+1) / 2} V_{n m+1}( \pm 2 \beta)$

By numerically solving Eq. (8) we obtain the eigenvalues $E_{n}\left(k_{y}\right)$ and eigenfunctions $C_{n}$. This nonperturbative approach is not suitable for very weak magnetic field since the concept of Landau orbit will fail if $\omega_{c} \tau<1$. So in the following calculation of magnetoresistance, we start from magnetic field strength $B=0.08 \mathrm{~T}$ and assume $\omega_{c} \tau \gg 1$. With the above procedure, a typical energy spectrum is calculated numerically under weak and strong modulation (Fig. 1). One can find that at weak modulation, the width of the modulation-broadened Landau bands is an oscillatory function of the band index $n$ [Fig. 1(a)]. However, for strong modulation [Fig. 1(b)], the energy spectrum is very complicated and the Landau subbands overlap strongly so that the flatband condition determining the period of the Weiss oscillation cannot be applied in a simple way in these cases.

\section{MAGNETORESISTANCE}

To calculate the magnetoresistance $\rho_{x x}$, we follow the formulation of Refs. 4 and 13, which is based on the general Liouville equation with dissipation included explicitly. Under the one-particle approximation, the conductivity tensor is given by

$$
\begin{aligned}
\sigma_{\mu \mu}= & \frac{e^{2}}{k_{B} T \Omega} \sum_{\xi} f_{\xi}\left(1-f_{\xi}\right) \tau\left(E_{\xi}\right)\left\langle\xi\left|v_{\mu}\right| \xi\right\rangle^{2} \\
& +\frac{e^{2}}{2 k_{B} T \Omega} \sum_{\xi, \xi^{\prime}} f_{\xi}\left(1-f_{\xi^{\prime}}\right) W_{\xi \xi^{\prime}}\left(a_{\mu}^{\xi}-a_{\mu}^{\xi^{\prime}}\right)^{2},
\end{aligned}
$$

where $\mu=x, y, \Omega$ is the volume of the system, $|\xi\rangle$ is the single electron state, $\tau\left(E_{\xi}\right) \approx \tau$ is the relaxation time, $f$ is the Fermi-Dirac function, $W_{\xi \xi^{\prime}}$ is the transition probability due to impurity scattering, and $a_{\mu}^{\xi}=\left\langle\xi\left|r_{\mu}\right| \xi\right\rangle$. This formula has been successfully applied to weak modulation by electric and magnetic field on 2DEG's (Refs. 5 and 3 and to strong 1D EMS. ${ }^{7}$ The first term in Eq. (10) describes the extended-state contribution that leads to Drude conductivity for freeelectron gas. The second term describes the localized-state contribution. For the geometry of our problem, the states along the $\hat{y}$ direction are extended but along $\hat{x}$ are localized. So the first term in Eq. (10) contributes to $\sigma_{y y}$ and the second to $\sigma_{x x}$. Since $\sigma_{x x}$ shows no noticeable oscillations and $\sigma_{y x}^{2} \gg \sigma_{x x} \sigma_{y y}$, we have $\rho_{x x} \approx \sigma_{y y} / \sigma_{y x}^{2} \approx \sigma_{y y} B^{2} / e^{2} N_{s}^{2}$ $\left(\omega_{c} \tau \gg 1\right)$. Therefore, the minima of $\sigma_{y y}$ coincide with those of $\rho_{x x}$.

In the folllowing magnetoresistance calculation, we evaluate the conductivity $\sigma_{y y}$ with the ad hoc assumption of 


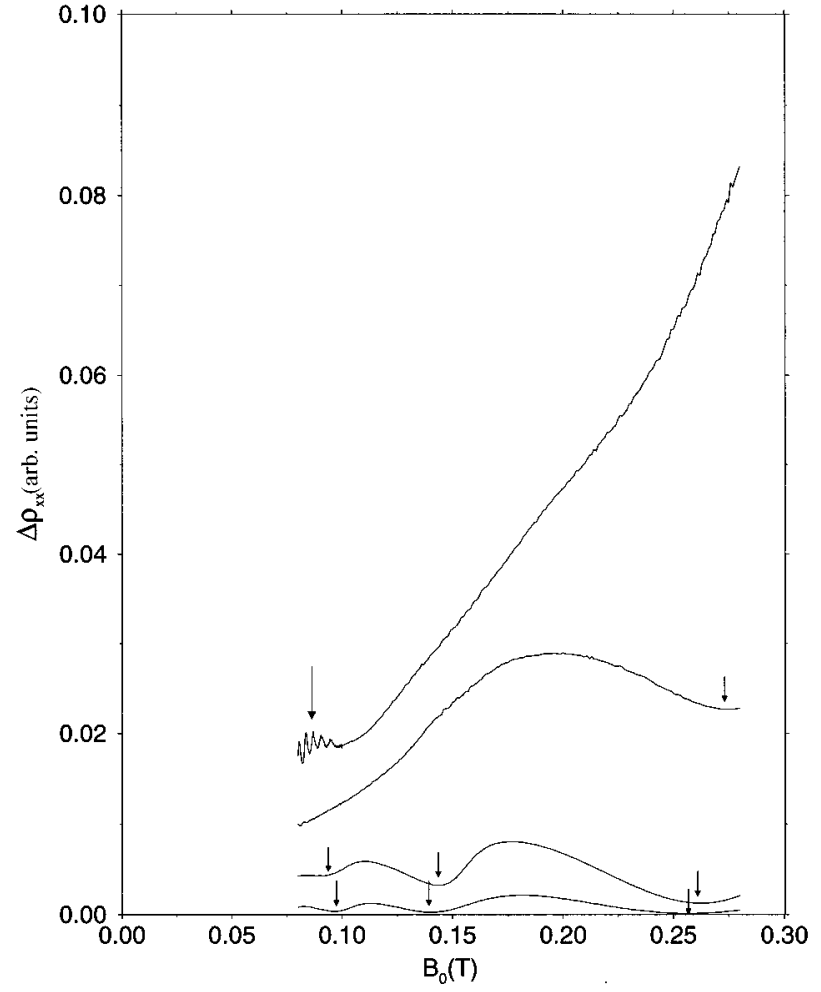

FIG. 2. Calculated magnetoresistance $\rho_{x x}$ vs magnetic field $B(T)$ at temperature $T=4.2 \mathrm{~K}$, spacing $a=500 \mathrm{~nm}$, area carrier density $n_{s}=2.2 \times 10^{11} \mathrm{~cm}^{-2}$, and various amplitude of periodic potential. $B_{1}=0.08 \mathrm{~T}, 0.16 \mathrm{~T}, 0.32 \mathrm{~T}, 0.48 \mathrm{~T}$ from lower to upper curve, respectively. The small arrows indicate the flatband situations defined by Eq. (2), where the band contribution has minima. The large arrow indicates the oscillations.

a constant relaxation time $\tau$. In the high mobility samples, the dominant contribution to magnetoresistance $\rho_{x x}$ comes from the Landau band electrons scattered by the disorder. So in the following calculation of magnetoresistance $\rho_{x x}$, we can neglect the contribution to magnetoresistance $\rho_{x x}$ due to the effect of the disorder-induced broadening of Landau band and use the quasiclassical (the constant $\tau$ ) approach. From this quasiclassical approach, we do not expect an exact quantitative agreement with the experimental results, but we can expect a qualitative understanding of the underlying physics.

With the first term of Eq. (10), we can calculate $\sigma_{y y}$ for any $B_{1}, a, v_{f}$, and $B_{0}\left(\omega_{c} \tau \gg 1\right)$. The matrix element of the velocity $\left\langle\xi\left|v_{x}\right| \xi\right\rangle$ can be determined by using the FeynmanHellman theorem,

$$
\left\langle n k_{y}, s\left|v_{x}\right| s, n k_{y}\right\rangle=\frac{1}{\hbar} \frac{d E_{n}\left(k_{y}\right)}{d k_{y}},
$$

where $|\xi\rangle=\left|n k_{y}, s\right\rangle$ and $s$ represents spin of electron. $E_{F}$ is determined self-consistently from

$$
N_{s}=2 \sum_{n k} f_{n k}
$$

where $N_{s}$ is the total number of electrons. In this paper, we use $B_{0}=0.08 \mathrm{~T} \sim 0.28 \mathrm{~T}, n_{s}=2.2 \times 10^{11}, a=500 \mathrm{~nm}$, and

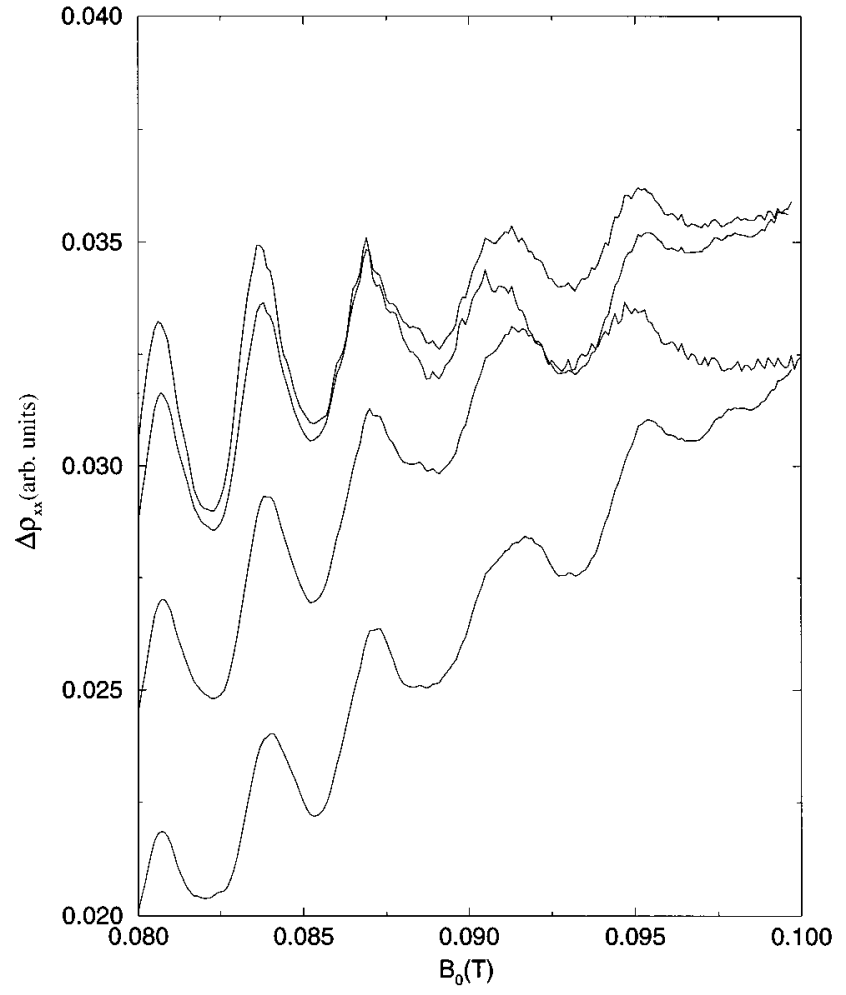

FIG. 3. Calculated magnetoresistance $\rho_{x x}$ at various temperatures of a 2 DEG subjected to a strong magnetic modulation field $B_{1}=0.48 \mathrm{~T}$ and a low uniform magnetic field $B_{0}=0.08 \mathrm{~T} \sim 0.10 \mathrm{~T}$. The curves correspond to temperature equal to $4.2 \mathrm{~K}$ (top), 10, 20, and $40 \mathrm{~K}$ (bottom).

$B_{1}$ from $0.08 \mathrm{~T}$ to $0.48 \mathrm{~T}$. These parameters include all the maxima or minima of the Weiss oscillations and give $E_{F} \sim 8 \mathrm{meV}$, which corresponds to filling up to about the sixtieth Landau level at $B_{0}=0.08 \mathrm{~T}$. The eigenvalues $E_{n}\left(k_{y}\right)$ and eigenstate are calculated by diagonalizing Eq. (8) for $C_{n}$, with $n=120$.

We now present our calculation of magnetoresistance for several parameters within experimental range. Figure 2 shows magnetoresistance curves calculated for electron density $n_{s}=2.2 \times 10^{11}$ corresponding to $E_{F}=8 \mathrm{meV}$, magnetic field strength $B_{0}=0.08 \quad \mathrm{~T} \sim 0.28 \quad \mathrm{~T}, \quad a=500 \mathrm{~nm}$, $m^{\star}=0.07 m_{e}$, and various values of $B_{1}$. In Fig. 2, one can see that for a weak potential, or small $\gamma / \beta$, our nonpertubative numerical calculations of magnetoresistance is very similar to the perturbative ones by Xue and $\mathrm{Xiao}^{3}$ and do reproduce the Weiss oscillation. The minima of $\rho_{x x}$ satisfies the flatband conditions obtained in perturbative calculation ${ }^{3,4}$ and the Weiss oscillation is due to the oscillatory linewidth of the modulation-broadened Landau bands. ${ }^{8}$ But as the magnetic modulation $B_{1}$ increases and $\gamma / \beta$ becomes larger, the extremum of Weiss oscillations gradually disappears and the magnetic modulation $B_{1}$ required to quench a particular ex tremum at a given uniform magnetic field $B_{0}$ increases with $B_{0}$. Physically, it is because different Landau bands hybridize strongly as the amplitude of the potential becomes larger, so that the feature of the oscillatory linewidth of the energy spectrum gradually disappears. This behavior is very similar to that found in the EMS case. However, when the magnetic 
modulation is increased further, Fig. 2 shows that the original Weiss oscillations which are determined by the MMS term are quenched, and a series of oscillations appears at the very low uniform magnetic field $(0.08 \mathrm{~T} \sim 0.10 \mathrm{~T})$. The minima and maxima of $\rho_{x x}$ of these new oscillations do not satisfy the Eq. (1) and Eq. (2). To understate the mechanism behind these new oscillations, we calculate the magnetoresistance at various temperatures shown as Fig. 3. One can see that these new oscillations persist to high temperature (at least $40 \mathrm{~K}$ ), although the amplitude of the oscillations is slowly decreasing as the temperature increases. At a finite temperature $T$, electrons within an energy $\sim k_{b} T$ in the neighborhood of the Fermi energy $E_{F}$ contribute to the electrical transport. The fact that these oscillations persist at higher temperature and their temperature dependence is very similar to that observed experimentally in the case of weak EMS (Ref. 14) suggest that the mechanism of these new oscillations, similar to the Weiss oscillations, is also due to the oscillatory linewidth of the modulation-broadened Landau bands. This is not a surprising result, since the EMS term takes a more important role which cooperates with the MMS term to produce the new oscillations.

In Fig. 2 and Fig. 3, we do not give the absolute value of $\rho_{x x}$ as it depends on the relaxation time $\tau$. This does not influence our qualitatively understanding of the MMS in 1D strong modulation. We believe that the nice qualitative agreement between the theoretical predictions and experimental results ${ }^{7}$ in 1D strong modulation of the EMS gives strong support to our theoretical results which describe the basic mechanism for the production and the quenching of the novel magnetoresistance oscillations.

\section{CONCLUSION}

In conclusion, we have presented a nonperturbative calculation of the magnetoresistance under 1D magnetic modulation at different strength. We not only generate results in agreement with the weak field experiment and predict the quenching of the Weiss oscillations at strong magnetic modulation, but we also find that the process of the quenching of the Weiss oscillation in the MMS case is very similar to that in the EMS case . Furthermore, we predict the appearance of a series of magnetoresistance oscillations. The temperature dependence of these oscillations suggests that their basic mechanism is similar to that of the Weiss oscillations. Indeed, perturbation calculation in our model produces similar results to the calculation of Xue and Xiao. ${ }^{3}$ However, the series of oscillation at strong modulation can only be found in the nonperturbative calculation. For future work, we would like to clarify the effect of disorder-induced broadening of the Landau band. It is well known that disorderinduced broadening of the mini-Landau band will smear out the oscillation linewidth of the energy spectrum. Nevertheless, we believe that a full treatment of the nonperturbative quantum-mechanical calculation of the quenching of the magnetoresistance oscillation, although neglecting the effect of disorder-induced broadening of the Landau band and multi-center scattering, does contain the basic mechanism of the magnetoresistance oscillations in the strong 1D MMS.

\section{ACKNOWLEDGMENTS}

The support of an RGC grant through HKUST is acknowledged. Discussions with P. Sheng, X. Yan, and Z. Zhang are acknowledged.
${ }^{1}$ P.D. Ye, D. Weiss, R.R. Gerhardts, M. Seeger, K. von Klitzing, K. Eberl, and H. Nickel, Phys. Rev. Lett. 74, 3013 (1995).

${ }^{2}$ H.A. Carmona, A.K. Geim, A. Nogaret, P.C. Main, T.J. Froster, M. Henini, S.P. Beaumont, and M.G. Blamire, Phys. Rev. Lett. 74, 3009 (1995).

${ }^{3}$ D.P. Xue and G. Xiao, Phys. Rev. B 45, 5986 (1992).

${ }^{4}$ F. M. Peeters and P. Vasilopoulos, Phys. Rev. B 47, 1466 (1993).

${ }^{5}$ R.R. Gerhardt, D. Weiss, and K. v. Klitzing, Phys. Rev. Lett. 62, 1173 (1989).

${ }^{6}$ P.H. Beton, E.S. Alves, P.C. Main, L. Eaves, M.W. Dellow, M. Henini, and O.H. Hughes, Phys. Rev. B 42, 9229 (1990).

${ }^{7}$ Q.W. Shi and K.Y. Szeto, Phys. Rev. B 53, 12990 (1996).
${ }^{8}$ D. Weiss et al., Europhys. Lett. 8, 179 (1989); Phys. Rev. B 39, 13020 (1996); High Magnetic Fields in Semiconductor Physics II, 1989, edited by G. Landwehr (Springer, Berlin, 1989), p. 357. ${ }^{9}$ C.W.J. Beenakker, Phys. Rev. Lett. 62, 2020 (1989).

${ }^{10}$ M. Abramowitz and I.A. Stegun, Handbook of Mathematical Functions (Dover, New York, 1964).

${ }^{11}$ I.S. Ibrahim and F.M. Peeters, Phys. Rev. B 52, 17321 (1995).

${ }^{12}$ X. Wu and S.E. Ulloa, Phys. Rev. B 47, 7182 (1993).

${ }^{13}$ M. Charbonneau et al., J. Math. Phys. 23, 318 (1982).

${ }^{14}$ P.H. Beton, P.C. Main, M. Dellow, R.P. Taylor, E.S. Alves, and L. Eaves, Phys. Rev. B 42, 9689 (1990). 